\title{
Quality of life of Elderly Diabetic and Hypertensive People - Impact of Intervention Programme
}

\author{
Roopa, K.S., and Rama Devi, G. \\ Department of Human Development and Research Centre, Smt. V.H.D. Central Institute of Home Science, \\ Bangalore University, Bangalore - 560001 , Karnataka, India,
}

\begin{abstract}
A research project was conducted to study the quality of life $(Q o L)$ of elderly diabetic and hypertensive people in Bangalore city. The objective of the study was to evaluate an educational module developed to help the elderly to cope with prevailing conditions and improve their quality of life. The sample consisted of 80 elderly of which 40 were men and 40 were women between the age group 65 to 76 years. The QoL was assessed through the standardized scale WHO -QoL-100 felid version. A quasi experimental approach with a repeated measure design was used with provision to compare the pre and post intervention assessment for the evaluation of the non-formal educational module for its effectiveness in improving the knowledge and quality of life of the elderly. The ' $t$ ' test was applied to see the significance of differences in the mean scores of men and women and the two age groups as well as the pre and post intervention assessments. The findings revealed that the post test scores on the overall $Q O L$ were higher than the pre test scores and the individual differences in the scores were also reduced considerably during post test for men and women as well as the two age groups. Statistically significant gain was noticed on all the five domains of QOL after the intervention program.
\end{abstract}

\section{Introduction}

Diabetes in the elderly is emerging as one of the most important public health problems of the $21 \mathrm{st}$ century. With increasing life expectancy, the numbers of older people are growing in our society. There are many illnesses that occur in association with age. Type-2 diabetes and hypertension is the classical example of diseases that increases with age. Changes in life style, including diet and physical activity, and the increasing numbers of elderly people are both key factors for the worldwide growth in the number of cases of diabetes.

Adults turning 65 this year can expect to live, on average, another 19 years, but for a significant part of their later years, many older people are living with serious health problems. Most have more than one. Data indicate that more than half have three or more chronic diseases and thereby meet the criteria for multi morbidity (AGS 2012).

Old age people are more prone to develop diabetes. Especially, due to overweight and lack of mobility they fall prey to type 2 diabetes mellitus. Glucose intolerance is a common occurrence among old age people. Most often it is difficult to identify symptoms of the disease in old people. However, it gets identified by other conditions like cardiovascular disease and the like.

Basic care for disease is the same as for all age people but old people have greater chances of developing complications. Biggest problem with old age people is the slow rate of weight reduction as they cannot go for rigorous exercises. Since exercise does not give good results, meal planning becomes more important for normalizing blood pressure and blood sugar level. Certain medicines are also not given to old age diabetics as it may harm them. Most often old people possess undiagnosed diabetes and it comes into light with severe complications like neuropathic foot lesions. So, old age people and particularly diabetics of that age must take good care of their foot. Elderly diabetics are vulnerable to complications to neuropathy, nephropathy, vascular disease and hypertension. So, a very restricted life from the point of view of medication and meal along with constant monitoring of blood glucose is must for them.

Diabetes is considered to be a life-threatening disease among the older people in the present century. Along with the problems of age, they also have to cope up with the effects of diabetes on this aging process, be it cardiovascular problems or Parkinson's disease. It is difficult indeed to shoulder such a responsibility at such an age. Diabetes is responsible for high mortality rate among the older people, particularly those at 65 years and above.

In the most general terms, quality of life may be thought of as a multidimensional construct incorporating an individual's subjective perception of physical, emotional, and social well-being, including both a cognitive component (satisfaction) and an emotional component (happiness). Quality of life is defined by WHO as "individuals perception of their position in life in the context of the culture and value system in which they live and in relation to their goals, expectation, standards and concerns" (WHO 1996). 
Quality of life is an important health outcome in its own right, representing the ultimate goal of all health interventions. Quality of life is measured as physical and social functioning, and perceived physical and mental well-being. People with diabetes may have a low level of quality of life than people with no chronic illness, but a better quality of life than people with most other serious chronic diseases. Duration and type of diabetes are not consistently associated with quality of life. Intensive treatment does not impair quality of life, and having better glycemic control is associated with better quality of life. Complications of diabetes are the most important disease-specific determinant of quality of life. Numerous demographic and psychosocial factors influence quality of life and should be controlled when comparing subgroups. Studies of clinical and educational interventions suggest that improving patient's health status and perceived ability to control their disease results in improved quality of life. Methodologically, it is important to use multidimensional assessments of quality of life, and to include both generic and disease-specific measures. Quality of life measures should be used to guide and evaluate treatment interventions.

In 1948 the World Health Organization defined health from a new perspective, stating that health was defined not only by the absence of disease and infirmity, but also by the presence of physical, mental and social well-being. In recent years, there has been a burgeoning interest in quality of life issues, and especially in health-related quality of life, fuelled by several factors, including a growing body of evidence concerning the potent effect of psychosocial factors on physical health outcomes, and dramatic changes in the organization and delivery of health care.

Recent developments in the fields of health outcome research and health technology assessment have also fuelled the tremendous increase in the use of quality of life evaluation as a technique for clinical research. Greater attention is now being devoted to evaluating the quality of health care and the economic value associated with new interventions. Managed care organizations have stimulated a growing effort to determine whether the costs associated with new or existing therapies and educational interventions are justified within fairly short time frames, often less than 3 years. As a consequence, researchers and health plan administrators are focusing on shorter-term patient outcomes, including functional health status, satisfaction with health care and overall quality of life. The goals of monitoring psychosocial well-being and quality of life in people with diabetes include: identification of people who are depressed or anxious; evaluating new treatments by identifying psychological costs and benefits; and identifying dissatisfaction with treatment and other aspects of care.

An attempt was made to develop a module of educational programme and an evaluation of this module as an intervention to improve the quality of life of elderly diabetic and hypertensive people.

\section{Objectives:}

1.To assess the quality of life (QOL) among old age people with diabetes and hypertension and to compare men and women as well as two age groups of elderly on QOL.

2.To assess and compare men and women as well as the two age groups of elderly with diabetes and hypertension on five dimensions of quality of life.

i. Physical health

ii. Psychological

iii. Level of Independence

iv. Social Relationship

v. Environment and over all perception of QOL

3.To develop a module of educational program for old age people for better management of the disease and to improve their health and quality of life.

4.To assess the impact of the intervention program (Application of the module of educational program developed in the study) on improving the knowledge, positive attitude in the management of the disease and on the overall perception of quality of life of the experimental

\section{Hypothesis} group of respondents.

1. There will be significant difference in the perception of quality of life of a). men and women, b). two age groups of elderly on the following dimensions:
i. Physical health
ii. Psychological
iii. Level of Independence
iv. Social Relationship
v. Environment and
vi. Over all perception of QOL

2. There will be a positive impact of educational program QOL of a). Men and women respondents and $b$ ). Two age groups of elderly. 


\section{Selection of Sample:}

Purposive random technique was used in the study. A total sample of 80 elderly who were diabetic and hypertensive people between the age group of 65 to 76 years of which 40 were men 40 were women. Further they were classified into $65-70$ and $70-76$ years.

Tool:

Standardized scale of Quality of life, WHO-QOL field version (1996) is the tool used in the study. This is a detailed comprehensive and holistic assessment scale focusing on the aspects of health and reliable international measure of QOL developed by World Health Organization. It consists of 100 questions split into five broad domains having twenty-three different facets.

The WHO QOL assessments have been found to allow detailed quality of life data to be gathered on a particular population, facilitating the understanding of diseases and the development of treatment methods. It is possible to carry out multi center quality of life research and compare results obtained in different field settings. The tool has been developed cross culturally and therefore is valid and the assessments are genuinely reliable and sensitive to any settings.

\section{Procedure:}

The study was conducted in Bangalore city. An assessment of quality of life elderly diabetic and hypertensive people was made by administering the WHO -QoL on a sample 80 elderly men and women who were willing to participate in the programme randomly selected from the area of Kumarapark, Sheshadripuram and Palace Guttahalli constituted the experimental group of respondents and they were subjected to participate in the educational program.

The module of educational program was designed by the investigators to cover the different aspects in the management of diabetes and hypertension and ways to improve health and quality of life. The program was scheduled for a period of two months consisting of eight sessions with four sessions per month, or one session per week of three hour's duration.

The program covered different areas viz., management of diabetes and hypertension, life style modification, food and nutrition, diet plan and counselling, stress management, acupressure, healthy and active aging, prevention of osteoporosis, health care and importance of physical exercises, laughter yoga and betterment of quality of life. Each session was conducted with a specific objective of providing relevant information. Various audio visual aids, lectures, demonstration, individual and group counselling, group activities, question and answer, summarizing the concept described, diabetes camp focusing on blood sugar and blood pressure test, bone mineral density test, eye test were organized for the benefit of participants and to improve their health. The impact of educational program was assessed using the same WHO-QOL with an interval two months between the intervention and re-assessment.

\section{Results and Discussion}

The mean scores and SD of scores on QOL for men and women are given in table -1. The ' $t$ ' values for significance of difference between the mean score of pre and post tests suggest that these differences are statistically significant accepting the hypothesis set for the study. The post test scores on QOL were higher than the pre test scores and individual differences in the scores were also reduced considerably during post test both for male and female respondents. Men compared to women showed a lower score on QOL during the pre test. However these differences were considerably reduced during the post test suggesting that the gain shown by men was more than the gain shown by women as indicated in the difference column.

Table - 1: Comparison of pre and post test Scores on overall perception of QOL of men and women respondents

\begin{tabular}{|l|l|l|l|l|l|l|l|l|}
\hline \multirow{3}{*}{ Gender } & \multirow{3}{*}{ Sample (n) } & \multicolumn{9}{|c|}{ Respondents Quality of Life } & \multirow{2}{*}{ Paired 't' } \\
\cline { 3 - 9 } & & \multicolumn{2}{|c|}{ Pre test } & \multicolumn{2}{|c|}{ Post test } & \multicolumn{3}{c|}{ Difference } \\
\cline { 3 - 9 } & & Mean & SD & Mean & SD & Mean & SD & \\
\hline Men & 40 & 60.72 & 9.4 & 78.97 & 5.6 & 18.25 & 10.9 & $10.59^{*}$ \\
\hline Women & 40 & 69.79 & 9.9 & 86.21 & 6.6 & 16.42 & 5.9 & $18.58^{*}$ \\
\hline Combined & 80 & 65.25 & 10.6 & 82.59 & 7.1 & 17.33 & 8.8 & $17.61^{*}$ \\
\hline
\end{tabular}

*Significant at $5 \%$ level

The pre and post tests means scores on overall perception of quality of life of men and women respondents is shown in figure -1 . 


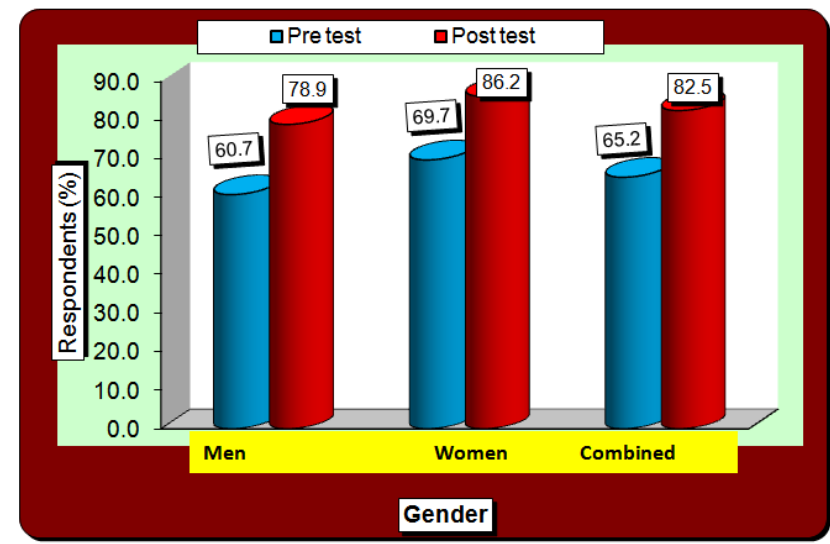

Figure -1: Comparison of pre and post test scores on overall perception of QoL of Men and Women respondents

Table - 2: Comparison of Pre and Post test Scores on Overall Perception of QOL of two Age groups of Respondents

\begin{tabular}{|c|c|c|c|c|c|c|c|c|}
\hline \multirow{3}{*}{ Age (years) } & \multirow{3}{*}{ Sample (n) } & \multicolumn{6}{|c|}{ Respondents Quality of Life } & \multirow{3}{*}{$\begin{array}{l}\text { Paired 't' } \\
\text { Test }\end{array}$} \\
\hline & & \multicolumn{2}{|c|}{ Pre test } & \multicolumn{2}{|c|}{ Post test } & \multicolumn{2}{|c|}{ Difference } & \\
\hline & & Mean & SD & Mean & SD & Mean & SD & \\
\hline $65-70$ & 50 & 65.85 & 11.7 & 84.72 & 7.6 & 18.87 & 8.0 & $16.68 *$ \\
\hline $71-76$ & 30 & 64.25 & 8.7 & 79.03 & 4.3 & 14.78 & 9.5 & $8.52 *$ \\
\hline Combined & 80 & 65.25 & 10.6 & 82.59 & 7.1 & 17.33 & 8.8 & $17.61 *$ \\
\hline
\end{tabular}

The mean scores and SD of scores on QOL for the two age groups are given in table -2. The ' $t$ ' values for significance of difference between the mean score of pre and post tests suggest that these differences are statistically significant. The post test scores on QOL were higher than the pre test scores and individual differences in the scores were also reduced considerably during post test for both the age groups of respondents. The gain in scores for the younger group among the aged was more than the gain for the older group of respondents. The pre and post test scores on overall perception QoL of two age group of respondents is shown in figure -2 .

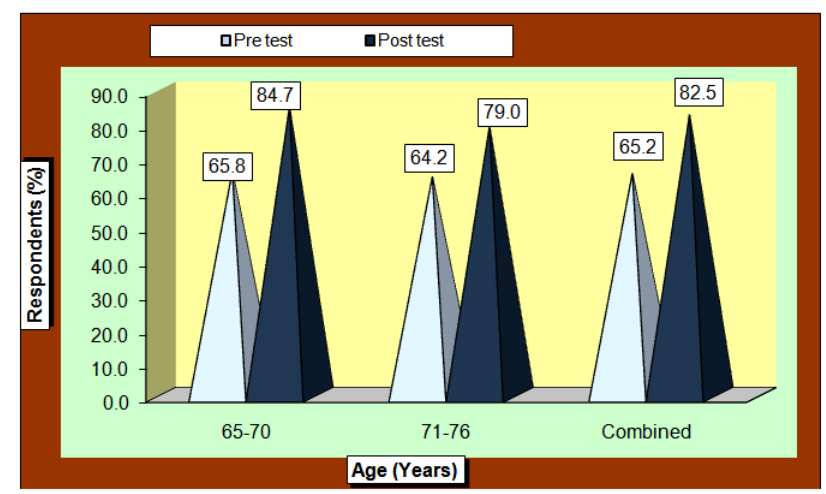

Figure - 2: Comparison of pre and post test scores on overall perception of QoL of two age groups respondents

Table - 3: Comparison of pre and post test scores of respondents on various domains of QOL

\begin{tabular}{|c|c|c|c|c|c|c|c|c|}
\hline \multirow{3}{*}{ No. } & \multirow{3}{*}{ Domains } & \multicolumn{6}{|c|}{ Respondents Quality of Life } & \multirow{3}{*}{$\begin{array}{l}\text { Paired 't' } \\
\text { Test }\end{array}$} \\
\hline & & \multicolumn{2}{|c|}{ Pre test } & \multicolumn{2}{|c|}{ Post test } & \multicolumn{2}{|c|}{ Difference } & \\
\hline & & Mean & SD & Mean & SD & Mean & SD & \\
\hline I & Physical & 62.50 & 8.9 & 80.57 & 8.8 & 18.07 & 7.2 & $22.45 *$ \\
\hline II & Psychological & 58.35 & 7.6 & 79.01 & 9.2 & 20.67 & 9.3 & $19.88 *$ \\
\hline III & Level of Independence & 65.84 & 12.4 & 81.43 & 7.0 & 15.58 & 11.0 & $12.67 *$ \\
\hline IV & Social Relationship & 68.96 & 13.0 & 85.06 & 7.9 & 16.10 & 11.8 & $12.20 *$ \\
\hline $\mathrm{V}$ & Environment & 71.27 & 15.6 & 87.41 & 7.9 & 15.14 & 15.1 & $8.97 *$ \\
\hline
\end{tabular}

*Significant at $5 \%$ level 
The mean scores and SD of scores on five domains of QOL for the experimental group of respondents are given in table -3 . The ' $t$ ' values for significance of difference between the mean score of pre and post tests suggest that these differences are statistically significant on all the domains. The post test scores on QOL were higher than the pre test scores and individual differences in the scores were also reduced considerably during post test except for physical and psychological domains. The results of pre and post test mean scores on different dimensions of QoL of respondents is shown in figure -3 .

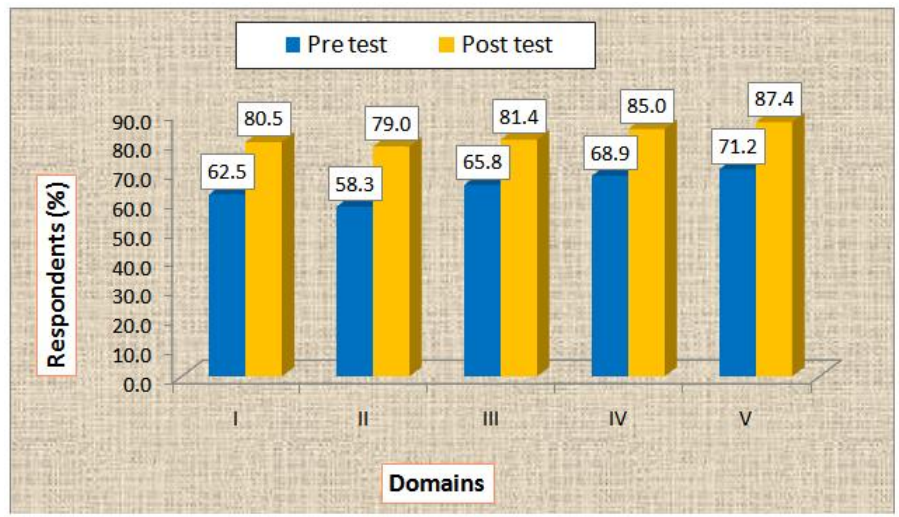

Figure-3: Comparison of pre and post test scores of respondents on various domains of QOL

The hypotheses, that there will be a positive impact of educational program on a).men and women respondents, and b).two age groups of old people with regard to QOL has been confirmed in this study.

In the present study knowledge was imported by organizing a educational program as an intervention for a group of 80 elderly with a module of program developed by the investigators . Various sessions were conducted by experts in the field; display of various charts, posters at the designated areas and distribution of booklets, leaflets followed by small group discussion. Since some of the elderly reported joint pains, back pain bone mineral test (BMD) test was also organized for them at free of cost. Some of them were diagnosed with calcium deficiency and free sample of calcium tablets were also distributed. Diabetic camp organized where blood sugar, blood pressure, skin sensation test, eye test was also conducted. This helped for the respondents showed keen interest in attending the program and get information relevant, realistic and acceptable for behavioral change. Scientific sessions were made more interesting with well chosen resource persons, visual aids, diet counseling that helped to create interest, hold attention and encourage learner participation.

Few sessions focused on life style modification, stress management, acupressure health care, importance of meditation physical exercise, laughter yoga to improve their quality of life.

Thus the post intervention results showed a significant gain in scores in quality of life in the experimental group of respondents suggesting the impact of educational program and its effectiveness is bringing about the desired change among respondents there by accepting the hypothesis set for the study.

Many myths and misconception about symptoms of old age and about diabetes were also noticed among respondents. They were made to clear their doubts.

In the present study it was noticed that all the participants participated in the program with keen interest. They also expressed that the program was unique and such programs are the need of the hour. They were of the opinion that elderly are neglected in the society.

Hypertension and diabetes mellitus presents a major area for intervention because it is a frequent condition and is amenable to control though both non pharmacological lifestyle factors and pharmacological treatment. Several models have been proposed to account for health behaviors and sustained behavioral changes. Although they may differ in content and perspective, models for behavior change stress the importance of evaluating the perceptions, attitudes, beliefs and outcome expectations of individuals as a crucial means to understand observed behaviors and to guide behavioral change.

Earlier studies have also shown that the first line of treatment for hypertension is the same as the recommended preventive lifestyle changes such as the dietary changes, physical exercise, and weight loss, can significantly reduce blood pressure in people with hypertension. If hypertension is high enough to justify immediate use of medications, lifestyle changes are still recommended in conjunction with medication. Dietary modification, weight control and regular exercise are the main approaches in the management of diabetes as well (Lteif et.al., 2005).

There is no single recommended dietary plan for people with diabetes. Rather, medical nutrition therapy for older people with diabetes should be individualized, with consideration given to usual eating habits and other lifestyle factors. The fundamental principle is to provide a nutritionally balanced diet. In general, 
normal body weight should be maintained. The normal body mass index of Indians needs to be determined since they develop diabetes and related illnesses at what is considered the normal range of BMI by the World Health Organization.

Physical exercise improves muscle strength and endurance and improves insulin sensitivity. Older people, unlike the young have to perform more regular and daily exercise to improve and sustain insulin sensitivity. Lifestyle modifications to prevent weight gain, especially abdominal accumulation of fat, are important for prevention and treatment of diabetes. Targets of glycemic control have to be determined with due consideration of age, remaining life-expectancy, co-morbid conditions and severity of vascular complications.

Physical activity participation is considered an ideal method by which blood pressure and blood sugar may be controlled. A substantial body of evidence exists for the physical and psychological health benefits that physical activity may confer to the older adult. For example, regular participation in moderate physical activity (such as walking) is associated with a reduced risk of falling, cardiovascular disease, type 2 diabetes mellitus (Mohan, Gokulakrishnan, Deepa, Shanthirani, and Datta, 2005).

Studies of clinical and educational interventions suggest that improving patient's health status and perceived ability to control their disease results in improved quality of life. Methodologically, it is important to use multidimensional assessments of quality of life, and to include both generic and disease-specific measures. Quality of life measures should be used to guide and evaluate treatment interventions.

Recent developments in the fields of health outcome research and health technology assessment have also fueled the tremendous increase in the use of quality of life evaluation as a technique for clinical research. Greater attention is now being devoted to evaluating the quality of health care and the economic value associated with new interventions. Managed care organizations have stimulated a growing effort to determine whether the costs associated with new or existing therapies and educational interventions are justified within fairly short time frames, often less than 3 years. As a consequence, researchers and health plan administrators are focusing on shorter-term patient outcomes, including functional health status, satisfaction with health care and overall quality of life. The goals of monitoring psychosocial well-being and quality of life in people with diabetes include: identification of people who are depressed or anxious; evaluating new treatments by identifying psychological costs and benefits; and identifying dissatisfaction with treatment and other aspects of care.

Quality of life may also be improved through physical activity participation in older adults, via increased bone health, functional capacity and independent living, as well as reduced depression, anxiety, and stress (Carr, 2001; Ministry of Health, 2003; National Health Committee, 1998).

The study on KAP of older Americans with hypertension supports the findings of Brent, M.E., et.al., (2003) that medications alone do not control blood pressure. Several life changes are required to lower blood pressure.

The population is ageing worldwide. Concurrently, diseases related to insufficient physical activity such as cardiovascular disease and Type 2 diabetes mellitus are increasing. Participation in regular, moderate intensity physical activity is related to a multitude of physical and psychological health benefits in older adults. Despite this, older adults are a population at risk of insufficient activity. Vigorous physical activity-activity that causes heavy sweating or large increases in heart rate - is better than moderate activity to maintain cardio respiratory fitness, but relatively few older persons engage in regular vigorous activity (i.e., three times per week or more), and the number declines steadily with age. Estimates for 2000 indicate that, only 13 percent individuals between the ages 65 and 74 reported engaging in vigorous physical activity for 20 minutes 3 or more days per week, and only $6 \%$ of those 75 and older reported such exercise (U.S.HHS,2000).

Therefore, effective intervention is essential to increase physical activity participation in the elderly. Elements related to intervention success with older adult populations have been reported extensively; however, limited information on the practical design and implementation of interventions and research is available.

Several prospective studies have shown that measures of lifestyle modification help in preventing the onset of diabetes. The Indian Diabetes Prevention Programme (IDPP), a preventive study done in India based on the Diabetes Prevention Program (DPP) has clearly documented the importance of physical activity in the prevention of diabetes. Early identification of the high risk individuals would help in taking appropriate intervention in the form of dietary changes and increasing physical activity, thus helping to prevent, or at least delay, the onset of diabetes. This means that identification of at risk individuals is extremely important if we are to prevent diabetes in India.

The most disturbing trend is the shift in age of onset of diabetes to a younger age in the recent years. This could have long lasting adverse effects on nation's health and economy. Early identification of at-risk individuals using simple screening tools like the Indian Diabetes Risk Score (IDRS) and appropriate lifestyle intervention would greatly help in preventing or postponing the onset of diabetes and thus reducing the burden on the community and the nation as a whole. 


\section{Conclusion}

The post test scores on the overall QOL were higher than the pre test scores and the individual differences in the scores were also reduced considerably during post test for men and women as well as the two age groups. The gain in score after intervention was more for men and the younger age group of aged. Statistically significant gain was noticed on all the five domains of QOL showed the impact of intervention program.

\section{Implications}

The study has for fetched implications since research pertaining to quality of life of diabetic and hypertensive elderly is limited in our country compared to western countries. The study provides insight into the areas knowledge, attitude and practices in the management of diabetes and hypertension and to improve their health and quality of life.

A module of educational program (user manual) is developed by the investigators. Practical suggestions were given by the experts in the specific fields for the respondents, consistent individual and group approaches using different techniques and media that appeal to the diabetic, enabled them to internalize and incorporate the ideas for betterment of perception of their overall quality of life.

The number of people with hypertension and diabetes mellitus is increasing due to population growth, aging, urbanization and increasing prevalence of obesity and physical inactivity. Quantifying the prevalence of hypertension and diabetes and the number of the elderly people affected with hypertension and diabetes mellitus, now and in the future, is important to allow rational planning and allocation of resources. The most viable and acceptable step would be to increase the awareness and preventive education in modifying the lifestyle of general population.

\section{References}

[1] American Geriatrics Society Expert Panel on the Care of Older Adults with Multi-morbidity. Guiding principles for the care of older adults with multi-morbidity: An approach for clinicians. Journal American Geriatric Society, Isuue-60, pp: E1-E25.

[2] Banegas,J.R., Lopez,G.E., Graciani,A., Guallar,C.P., Gutierrez,F.J.L., Alonso,J., and Rodriguez.A.F. (2007). Relationship between obesity, hypertension and diabetes, and health related quality of life among the elderly. European journal of cardio vascular prevention and rehabilitation Vol- 14(3), 456-462.

[3] Burka,V., Beilin,L.J., Grosskopf,S., Ritchie,J., Puddy,I.B., Rogers,P. (1992). Association of Life Style and Personality Characteristics with Blood Pressure and Hypertension: A Cross-Sectional Study In The Elderly, Journal of Clinical Epidemiology. Perth, Australia, Vol-45(10), pp: 1061-1070.

[4] Carr, H. (2001). Physical Activity and Health. The benefits of physical activity on minimising risk of disease and reducing disease morbidity and mortality: report. Wellington, NZ: Hillary Commission for Sport, Fitness and Leisure.

[5] Fujioka,Y., Ikegami,H., Fukuda,M., Kawaguehi.Y., Takegawa,K., Fujisawa,T., Ueda,H., Miki,T., Kumahara,Y., and Ogihara,T. (1993).The effect on age for the relationship between hypertension and glucose intolerance .Japanese journal of geriatrics , Vol-30 ,pp:622-625.

[6] James,E.G., Diane,G.S., Glenn,V.O., Soham,A.S., Kristen Peek., Kyriakos,M. ,and Kenneth,J.O.(2007). Health related quality of life in older Mexican Americans with diabetes: a cross-sectional study .Journal of health and quality of life outcome, pp: 5-39.

[7] Liisa Hittunen . (1999). Does glucose tolerance affect quality of life in an elderly population .Journal of diabetes research and clinical practice, Vol- 46,Issue 2,161-167.

[8] Lteif, A, Han,K, Mather.K.J., (2005). Obesity, Insulin Resistance and Metabolic Syndrome. Diabetes care, Vol- 16, pp:434-440.

[9] Ministry of Health. (2003). Population Ageing and Health Spending: 50-Year Projections. Wellington, New Zealand.

[10] Mohan ,V., Gokulakrishnan, K., Deepa, R., Shanthirani, C.S., Datta, M., (2005). Association of physical inactivity with components of metabolic syndrome and coronary artery disease - The Chennai Urban Population Study (CUPS No. 15). Diabetic Med, Vol--22, pp: 1206-11.

[11] National Health Committee. (1998). Active for Life: A call for action: the health benefits of physical activity. Wellington, NZ.

[12] Wandell, P.E and Jonas, T. (2000).The Quality of Life Of Elderly Diabetes Patients. Journal of Diabetes and Its Complications.

[13] WHO-QOL Group (1996). Field trial WHOQOL-100, Fact, Definitions and Questions, Geneva. 